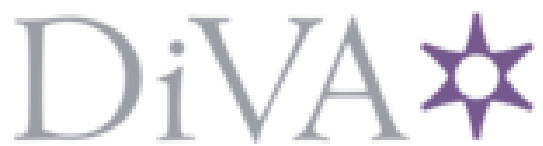

http://www.diva-portal.org

This is the published version of a paper published in .

Citation for the original published paper (version of record):

Mamani-Ortiz, Y., Marcela, L L., Ximena, A C., Elving, I V. (2020)

Diferencias de género en la interacción entre consumo de tabaco y alcohol con la presión arterial elevada

Gaceta Medica Boliviana, 43(2): 127-136

https://doi.org/10.47993/gmb.v43i2.189

Access to the published version may require subscription.

N.B. When citing this work, cite the original published paper.

Esta obra está bajo una licencia internacional Creative Commons Atribución-NoComercialCompartirIgual 4.o. https://creativecommons.org/licenses/by-nc-sa/4.o/

Permanent link to this version:

http://urn.kb.se/resolve?urn=urn:nbn:se:umu:diva-184058 


\title{
Diferencias de género en la interacción entre consumo de tabaco y alcohol con la presión arterial elevada
}

\author{
Gender differences in the interaction between tobacco and alcohol consumption with raised blood \\ pressure.
}

\author{
Mamani Ortiz Yercin ${ }^{1,2}$, Luizaga López Jenny Marcela ${ }^{2}$, Armaza Céspedes Ada Ximena ${ }^{2}$, Illanes Velarde Daniel Elving ${ }^{2}$
}

\section{Resumen}

\begin{abstract}
Objetivo: analizar las diferencias de género en la interacción entre consumo de tabaco y alcohol con la presión arterial elevada (PAE) en Cochabamba, Bolivia. Métodos: estudio transversal, con $n=10704$ participantes, seleccionados aleatoriamente. Se calcularon proporciones, Odds-ratios y análisis robusto de descomposición Blinder-Oaxaca. Resultados: $33,5 \%$ reportó que solo bebe, 1,8\% solo fuma y 9,2\% ambos. La probabilidad de presentar PAE fueron mayores en aquellos que fuman $(\mathrm{OR}: 2,04)$; beben y fuman $(\mathrm{OR}: 1,73) \mathrm{O}$ solo beben (OR:1,43). Los hombres presentaron prevalencias más elevadas de PAE, tabaquismo y consumo nocivo de alcohol; pero las mujeres que fuman presentaron niveles de OR más altos para el desarrollo de PAE. El nivel educativo, la edad, y el tipo de trabajo contribuyeron de manera positiva a explicar la brecha entre hombres y mujeres. Conclusión: la probabilidad de presentar PAE fue mayor en mujeres, especialmente en aquellas que fuman, a pesar de su baja prevalencia.
\end{abstract}

Palabras clave: tabaco, alcohol, presión arterial elevada, género, Bolivia

\section{Abstract}

Objective: to analyze gender differences in the interaction between tobacco and alcohol consumption with high blood pressure (HBP) in Cochabamba, Bolivia. Methods: a cross-sectional study was conducted, with $\mathrm{n}=10704$ participants, randomly selected. Proportions, Odds-ratios and robust analysis of Blinder-Oaxaca decomposition were calculated. Results. $33.5 \%$ of participants only drink alcohol, $1.8 \%$ only smoke and $9.2 \%$ both simultaneously. The probability of presenting HBP was higher in smokers (OR: 2.04); those who consume both simultaneously (OR: 1.73) or only drink alcohol (OR: 1.43). The men had a higher prevalence of HBP, smoking and harmful alcohol consumption; but women who smoke had higher OR levels to develop HBP. The differences in educational level, age, and type of work contributed positively to explain the gap between men and women. Conclusion. the probability of presenting HBP was higher in women, especially those who smoke, despite their low prevalence.

Keywords: tobacco, alcohol, high blood pressure, gender, Bolivia

$\mathrm{E}_{\mathrm{f}}^{\mathrm{l}}$ consumo nocivo de tabaco y alcohol son los principales factores de riesgo asociados a las enfermedades no transmisibles (ENT) en especial las enfermedades cardiovasculares (ECV) como la hipertensión arterial ${ }^{1-4}$. Si bien existen muchos estudios que analizan esta interacción, la mayoría evalúa su asociación por separado y se conoce muy poco sobre el efecto combinado de ambos ${ }^{2,4}$, a pesar de que su consumo generalmente se encuentra interrelacionado.

La mayoría de los estudios muestran diferencias significativas entre hombres y mujeres ${ }^{1,3}$; sin embargo, aún no se tiene claro las contribuciones de las diferencias sociales que implican vulnerabilidades o niveles de riesgo relacionados con la construcción y evolución de los roles sociales de género ${ }^{5}$, que determinan formas diferentes de exposición frente al tabaquismo y alcoholismo; por lo que cabe esperar que tengan un efecto diferente en el desarrollo de la hipertensión arterial.

\footnotetext{
${ }^{1}$ Instituto de Investigaciones Biomédicas e Investigación Social, Facultad de Medicina, Universidad Mayor de San Simón, Bolivia. https://orcid.org/0000-0003-0400-0414. ${ }^{2}$ Departamento de Epidemiología y Salud Global, Medicina, Facultad de Universidad de Umea, Suecia. https://orcid.org/0000-0003-2742-2994,

https://orcid.org/0000-0001-8602-8570, https://orcid.org/0000-0002-1458-8541. ${ }^{\star}$ Correspondencia a: Yercin Mamani Ortiz

Correo electrónico: yercin2003@hotmail.com

Recibido el 30 de septiembre de 2020. Aceptado el 24 de noviembre de 2020.
}

Según la Organización Mundial de la Salud (OMS), en todo el mundo $34 \%$ de los hombres y $6 \%$ de las mujeres mayores de 15 años eran fumadores para 2016, siendo más frecuente en la región europea (21\%) seguido por Latino América $(12 \%)^{6}$ donde se estima que cerca de 1 millón de personas mueren por causas relacionadas al tabaco y la exposición pasiva al humo de tabaco ${ }^{1}$. Similar situación es reportada para el consumo nocivo de alcohol, relacionada con más de 3,3 millones de muertes en el mundo ${ }^{6}$, y en el caso Latino Americano el consumo per cápita reportado fue de 6,9 L $\mathrm{L}^{3}$. Se estima que el $22 \%$ de los consumidores tienen episodios de consumo excesivo; $8 \%$ de los hombre y $3,2 \%$ de las mujeres llegan a desarrollar trastornos por abuso de alcohol según la Organización Panamericana de la Salud (OPS) ${ }^{1}$.

Las guías de manejo y tratamiento de Hipertensión Arterial remarcan el consumo de tabaco y alcohol como factores de riesgo modificables en el desarrollo de enfermedad cardiovascular ${ }^{7-10}$. Por el otro lado, los reportes de la OMS y OPS reflejan que los hombres presentan una mayor prevalencia de hipertensión arterial y otras $\mathrm{ECV}^{7}$. La carga de mortalidad asociada a ECV son mayores en hombres y aún mucho mayor en países de bajos y medios ingresos económicos ${ }^{1}$.

Un análisis sobre los factores que contribuyen a estas 
desigualdades puede mostrarnos un panorama más completo para el desarrollo de estrategias de promoción y prevención de la Hipertensión Arterial, focalizando nuestros esfuerzos en aquellas condiciones que contribuyen de manera significativa en el consumo de tabaco, alcohol y su repercusión cardiovascular. Desde un enfoque cuantitativo, la descomposición de Blinder-Oaxaca ${ }^{11,12}$ ha sido recientemente sugerida y ejemplificada como un método apropiado para clarificar el papel de los indicadores que representan los procesos sociales que sustentan las desigualdades en salud. Este enfoque de análisis busca explicar la distribución del resultado, en este caso la diferencia de género en la prevalencia de presión arterial elevada (PAE), consumo de tabaco y alcohol; entre grupos dominantes (hombres) y subordinados (mujeres) dentro la estructura social boliviana, a través de un conjunto de factores explicativos que varían sistemáticamente por las diferencias sociales entre ambos géneros.

El presente estudio tiene como objetivo: a) analizar las diferencias de género en la interacción entre el tabaquismo y consumo nocivo de alcohol con la PAE y b) determinar las contribuciones de los factores sociodemográficos y conductuales en las desigualdades de prevalencia entre hombres y mujeres de Cochabamba, Bolivia.

\section{Material y métodos}

\section{Contexto del estudio}

El presente trabajo es el tercer reporte de la "Encuesta Departamental sobre Factores de Riesgo Asociados a Hipertensión y Obesidad" (FRAHO), implementada de manera conjunta por el Instituto de investigaciones Biomédicas e Investigación Social de la Universidad Mayor de San Simón (IIBISMED-UMSS) y el Servicio Departamental de Salud (SEDES) del departamento de Cochabamba, Bolivia.

Diseño, muestra analítica y metodología de muestreo

En este artículo, se presentan los resultados de un sub proyecto de FRAHO, correspondiente a un estudio observacional, analítico, de corte transversal; implementada en todos los municipios $(\mathrm{N}=47)$ de Cochabamba; durante el periodo comprendido entre julio de 2015 a noviembre de 2016, utilizando la metodología WHO-STEPS para la vigilancia de factores de riesgo asociados a $\mathrm{ENT}^{13}$.

El tamaño de la muestra $(\mathrm{n}=10$ 609) se calculó sobre la base de estimaciones previas de la prevalencia de sobrepeso y obesidad en el departamento (alrededor del 30\%) utilizando un nivel de confianza del 95\%, un margen de error de 0,05; y un efecto de diseño de 1.05 según lo recomendado por el manual STEPS $^{7}$, con una asignación proporcional entre hombres y mujeres, para una población de $\mathrm{N}=1^{\prime} 240^{\prime} 771$ sujetos mayores de 18 años estimado para el 2015 por el Instituto Nacional de Estadística de Bolivia ${ }^{14}$.

Todos los sujetos fueron seleccionados mediante un muestreo aleatorio trietápico: a) Se seleccionaron 394 centros de atención primaria en salud (CAPS) que conformaron las unidades poblacionales de muestreo (UPM); b) en cada UPM se seleccionaron viviendas en base a la técnica de monitoreo rápido de coberturas de atención del Ministerio de Salud de Bolivia, adecuada para fines del estudio, seleccionando aleatoriamente 3 a 5 comunidades o barrios por cada CAPS ; c) en cada barrio o comunidad, se eligieron los domicilios con personas que cumplan los criterios de inclusión y en cada una se seleccionó a un individuo de 18 años o más, utilizando el método aleatorio de Kish recomendado en el manual de WHO-STEP ${ }^{13}$.

Se incluyeron a todas las personas mayores de 18 años con residencia por al menos 6 meses en el área de estudio. Se excluyeron a las mujeres embarazadas y aquellos que no dieron su consentimiento o con información parcial sobre las variables de evaluación. Un total de 12527 personas fueron seleccionadas, de las cuales el $85,45 \%$ aceptaron participar en el estudio y la muestra final utilizada para el análisis estadístico en este subproyecto fue de $n=10,704$.

\section{Recolección de la información y mediciones}

El procedimiento se basó en la versión Panamericana (V2.0) de la metodología STEPS adaptado al contexto boliviano por el equipo de investigación. Esta metodología consiste en 3 etapas $^{13}$ : a) El PASO 1 utiliza un cuestionario para recopilar datos sociodemográficos y de hábitos de vida o factores de riesgo conductuales. b) El PASO 2 involucra la evaluación y cálculo de indicadores antropométricos y de la presión arterial. c) El PASO 3 implica la evaluación y cálculo de indicadores laboratoriales del perfil metabólico; este paso no fue implementado en todo el departamento y no es parte de este reporte.

Las mediciones se realizaron mediante el uso de instrumentos calibrados y estandarizados en cada CAPS y las revisiones directas por personal de salud de las CAPS previamente capacitados en el desarrollo de habilidades para entrevistas y visitas de campo.

Indicador de resultado de salud: Presión arterial elevada.

La presión arterial se midió en ambos brazos después de un descanso de 5 minutos. Se realizó una tercera lectura si hubo una diferencia de más de $25 \mathrm{~mm} \mathrm{Hg}$ para la presión arterial sistólica o $15 \mathrm{~mm} \mathrm{Hg}$ para la presión arterial diastólica. Se utilizó la media de todas las medidas, con base en las recomendaciones del protocolo de la metodología STEPS. La presión arterial elevada se definió como una presión arterial sistólica de $\geq 130 \mathrm{~mm} / \mathrm{Hg}$ o una presión arterial diastólica de $\geq 85 \mathrm{~mm} / \mathrm{Hg}$ o el uso auto informado de medicamentos antihipertensivos, según las pautas de la OMS y de la Sociedad Americana de Cardiología para el $2015^{7}$.

Factor de exposición para la desigualdad en salud: referencia de género.

La identidad de género se basó en la información recopilada por la encuesta, incluyendo las categorías mujeres y hombres por autoidentificación individual durante la recolección de la información.

Factores explicativos de la desigualdad:

Los factores explicativos de las desigualdades de salud incluyeron variables con vínculos conocidos o posibles con la 
PAE y las desigualdades de género y se agruparon en:

1) Factores sociodemográficos:

a) Edad clasificada en cuatro grupos según lo recomendado en el manual STEPS: 18-29, 30-44, 45-59 y $\geq 60$ años.

b) Lugar de residencia clasificado según las 5 regiones sociodemográficas de Cochabamba: Andina, Cono Sur, Valle Central, Trópico y Valle Alto.

c) Estado civil categorizado como: soltero o que nunca se casó, actualmente casado, unión libre y viudo o separado.

a) Nivel de educación, clasificado en cuatro grupos: No recibió educación formal, educación primaria, secundaria y superior.

b) Ocupación clasificada en seis grupos: estudiantes, trabajadores por cuenta propia, empleados, labores de hogar, jubilados y desempleados.

2) Factores de riesgo de comportamiento:

a) Para el tabaquismo, se clasificó los sujetos como fumador actual o no fumador de acuerdo con el manual de la encuesta de WHO-STEPS.

b) El consumo de alcohol se exploró a través de los ítems adoptados de la "Prueba de identificación de trastornos por uso de alcohol (AUDIT)" incluidos en la encuesta STEPS, que recopilan información sobre tres aspectos diferentes: cantidad, frecuencia y patrones de consumo. Los participantes se clasificaron en la categoría de uso nocivo de alcohol ( $1=$ presente; $0=$ ausente) si se informaron episodios de consumo excesivo de alcohol (6 o más bebidas estándar cada vez que consume bebidas alcohólicas): a) una o más veces al mes en los últimos 12 meses; b) dos veces o más en los últimos 30 días; o c) si bebió hasta perder el conocimiento al menos una vez en los últimos 7 días.

\section{Análisis estadístico}

Los datos se ingresaron en una base de datos en Microsoft Excel $^{\circledR}$; posteriormente se realizó la limpieza de sujetos con información faltante y el análisis de datos utilizando STATA v15.0 (StataCorp LLC-U.S.A.). La normalidad de las variables cuantitativas fue verificada mediante la prueba de Kolmogorov-Smirnov ${ }^{15}$. El análisis descriptivo se realizó en forma de proporciones. Para la estimación del riesgo de PAE por consumos de tabaco y/o alcohol, se calcularon Odds-Ratios (OR) crudos y ajustados a través de modelos de regresión logística binaria y multivariada.

Para el análisis de descomposición de las desigualdades entre hombres y mujeres para la PAE, así como el consumo de tabaco y alcohol se utilizó la versión robusta del método de Blinder-Oaxaca para resultados binarios ampliamente descritos por Jann ${ }^{12}$, que se basa en dos modelos de regresión logística que se ajustan por separado para cada uno de los grupos y luego divide la brecha de salud entre los grupos en una fracción atribuible a las diferencias en los factores explicativos (la parte explicada) ya las diferencias en coeficientes (la parte no explicada). Para fines prácticos, los factores se agruparon en 2 dominios: factores sociodemográficos (grupo etario, etnicidad, residencia, nivel de educación, estado civil y ocupación), y los factores de riesgo de comportamiento (tabaquismo y consumo del alcohol). La parte total explicada y no explicada, así como la contribución independiente de cada uno de los factores explicativos, se informan como contribuciones absolutas (en la misma escala que el resultado) y como contribuciones relativas (porcentajes).

Consideraciones éticas.

El proyecto marco FRAHO, contó son la aprobación del comité de ética de la Facultad de Medicina de la Universidad Mayor de San Simón en Cochabamba. Todos los participantes firmaron un consentimiento informado basado en el formulario de consentimiento de la metodología STEPS. Los datos recolectados fueron codificados y administrados exclusivamente por el equipo de investigación. Los participantes con PAE o cualquier otra enfermedad fueron remitidos al centro de salud más cercano para complementar su diagnóstico y tratamiento.

\section{Resultados}

Se analizo la información recabada de 10704 personas con una media de edad de $38 \pm 18$ años, el $57,38 \%$ fueron mujeres y la media de edad no tuvo diferencias estadísticamente significativas ( $\mathrm{p}>0,05)$ entre hombres $(39 \pm 19$ años) y mujeres (37 \pm 18 años).

\section{Tabaquismo}

La tabla 1 describe las características sobre el consumo de tabaco. La prevalencia global de tabaquismo (uso actual) fue de $11,1 \%$, siendo mayor en hombres $(21,6 \%)$. La experimentación del uso de tabaco también fue mayor en hombres (35,7\%). La

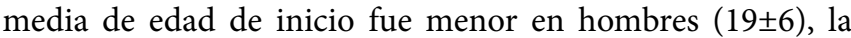
edad de abandono del hábito de fumar fue menor en mujeres $(26 \pm 11)$. Entre las personas que fuman actualmente, la media de cigarrillos consumidos fue mayor en hombres tanto por día $(0,42 \pm 1,5)$ y semana $(2,5 \pm 9,5)$ y las mujeres reportaron haber recibido consejo para dejarlo $(47,0 \%)$ o trataron de dejar de fumar $(45,5 \%)$ en mayor proporción de los hombres.

\section{Consumo de alcohol}

La tabla 1, tambien desglosa las características del consumo de alcohol desagregado por género, resaltando diferencias estadísticamente significativas entre hombres y mujeres para todas las variables evaluadas. El 66,5\% de los hombres y $45,9 \%$ de las mujeres reportaron haber consumido bebidas alcohólicas en algún momento de su vida, con una media de edad de inicio más temprano en los hombres (17士7). El 51,7\% recibió consejo para dejarlo, pero solo el 19,6\% intentó dejar su consumo.

La prevalencia de consumo actual de alcohol fue de $54,7 \%$ en los hombres y $33,9 \%$ en las mujeres. El 71,7\% de las mujeres reportó consumir con una frecuencia menor a una vez por mes; a diferencia de los hombres donde cerca del $50 \%$ reportó consumir por más de 1 vez al mes. El consumo mínimo por cada evento fue más frecuente en las mujeres $(65,5 \%)$. La prevalencia de consumo excesivo (hasta perder el conocimiento) por más de 6 veces al año fue mayor en hombres $(54,1 \%)$. 
Tabla 1: Prevalencia y caracterización del consumo de tabaco y alcohol

\begin{tabular}{|c|c|c|c|c|}
\hline \multicolumn{5}{|c|}{ CONSUMO DE TABACO } \\
\hline & & Mujeres & Varones & Global \\
\hline Edad (años) & & $37 \pm 18$ & $39 \pm 19$ & $38 \pm 18$ \\
\hline \multicolumn{5}{|l|}{ Consumió alguna vez } \\
\hline & No & $5672(92,3)$ & $2934(64,3)$ & $8606(80,4)$ \\
\hline & $\mathrm{Si}$ & $471(7,7)$ & $1627(35,7)$ & $2098(19,6)$ \\
\hline \multicolumn{5}{|l|}{ Uso activo } \\
\hline Edad de Inicio & & $21 \pm 8$ & $19 \pm 6$ & $20 \pm 6$ \\
\hline Le aconsejaron dejarlo & & $165(35,0)$ & $620(38,1)$ & $785(37,4)$ \\
\hline Trató dejararlo & & $176(37,4)$ & $673(41,4)$ & $849(40,5)$ \\
\hline \multirow[t]{3}{*}{ Edad a la que dejó de consu } & umir & $26 \pm 11$ & $31 \pm 13$ & $30 \pm 13$ \\
\hline & Tiempo (años) & $10 \pm 12$ & $11 \pm 12$ & $11 \pm 12$ \\
\hline & Tiempo (meses) & $3 \pm 3$ & $4 \pm 3$ & $4 \pm 3$ \\
\hline \multicolumn{5}{|l|}{ Uso actual } \\
\hline & No & $5943(96,7)$ & $3577(78,4)$ & $9520(88,9)$ \\
\hline & $\mathrm{Si}$ & $200(3,3)$ & $984(21,6)$ & $1184(11,1)$ \\
\hline & Le aconcejaron dejarlo & $94(47,0)$ & $425(43,2)$ & $519(43,8)$ \\
\hline & Trató dejarlo & $91(45,5)$ & $407(41,4)$ & $498(42,1)$ \\
\hline \multicolumn{5}{|c|}{ CONSUMO DE ALCOHOL } \\
\hline Edad (años) & & $37 \pm 18$ & $39 \pm 19$ & $38 \pm 18$ \\
\hline \multicolumn{5}{|l|}{ Consumió alguna vez } \\
\hline & No & $3324(54,1)$ & $1526(33,5)$ & $4850(45,3)$ \\
\hline & $\mathrm{Si}$ & $2819(45,9)$ & $3035(66,5)$ & $5854(54,7)$ \\
\hline \multicolumn{5}{|l|}{ Consumidores } \\
\hline Edad de Inicio & & $18 \pm 5$ & $17 \pm 7$ & $17 \pm 6$ \\
\hline Le aconsejaron dejarlo & & $1059(50,9)$ & $1307(52,4)$ & $2366(51,7)$ \\
\hline Trató de dejarlo & & $525(18,6)$ & $622(20,5)$ & $1147(19,6)$ \\
\hline \multicolumn{5}{|l|}{ Uso actual } \\
\hline & No & $4061(66,1)$ & $2065(45,3)$ & $6126(57,2)$ \\
\hline & $\mathrm{Si}$ & $2082(33,9)$ & $2496(54,7)$ & $4578(42,8)$ \\
\hline \multicolumn{5}{|c|}{ Frecuencia de consumo actual } \\
\hline & Todos los días & $13(0,6)$ & $35(1,4)$ & $48(1,0)$ \\
\hline & 5 a 6 días por semana & $21(1,0)$ & $45(1,8)$ & $66(1,4)$ \\
\hline & 3 y 4 días por semana & $0(0,0)$ & $1(0,0)$ & $1(0,0)$ \\
\hline & 1 y 2 días por semana & $98(4,7)$ & $279(11,2)$ & $377(8,2)$ \\
\hline & 1 y 3 días por mes & $436(20,9)$ & $823(33,0)$ & $1259(27,5)$ \\
\hline & $<1$ una vez al mes & $1493(71,7)$ & $1264(50,6)$ & $2757(60,2)$ \\
\hline \multicolumn{5}{|c|}{ Cantidad promedio de consumo } \\
\hline & Mínimo (1 a 3 porciones) & $1363(65,5)$ & $635(25,4)$ & $1601(35,0)$ \\
\hline & Medio (4 a 5 porciones) & $444(21,3)$ & $689(27,6)$ & $1222(26,7)$ \\
\hline & Elevado ( $>5$ porciones $)$ & $261(12,5)$ & $1171(46,9)$ & $1752(38,3)$ \\
\hline & No Responde & $2(0,1)$ & $1(0,0)$ & $3(0,1)$ \\
\hline \multicolumn{5}{|c|}{ Frecuencia de consumo excesivo } \\
\hline & 1 a 6 por año & $1363(65,5)$ & $1130(45,3)$ & $2493(54,5)$ \\
\hline & 6 a 12 por año & $444(21,3)$ & $706(28,3)$ & $1150(25,1)$ \\
\hline & $>12$ por año & $261(12,5)$ & $645(25,8)$ & $906(19,8)$ \\
\hline & No Responde & $14(0,7)$ & $15(0,6)$ & $29(0,6)$ \\
\hline \multicolumn{5}{|c|}{ Tipo de bebida más frecuente } \\
\hline & Artesanales fermentadas & $1127(54,1)$ & $1287(51,6)$ & $2414(52,7)$ \\
\hline & Cerveza & $759(36,5)$ & $1001(40,1)$ & $1760(38,4)$ \\
\hline & Destiladas & $117(5,6)$ & $146(5,8)$ & $263((5,7)$ \\
\hline & Otro tipo de bebidas & $62(3,0)$ & $43(1,7)$ & $105(2,3)$ \\
\hline & No Responde & $14(0,7)$ & $10(0,4)$ & $24(0,5)$ \\
\hline & Alcohol potable & $3(0,1)$ & $9(0,4)$ & $12(0,3 \%)$ \\
\hline
\end{tabular}

Significancia estadística calculado por T de Student para variables cuantitativas y X2 para las variables categóric
El tipo de bebida alcohólica consumida con mayor frecuencia fueron las bebidas artesanales (chicha, guarapo, garapiña, guindal o similares) tanto en hombres $(54,1 \%)$ como en mujeres (51,6\%).

\section{Uso combinado de tabaco y alcohol}

La tabla 2 muestra la distribución del consumo aislado o combinado de tabaco y alcohol, desagregado por género y las variables sociodemográficas evaluadas en la metodología WHO-STEPS.

El 55,4\% de la población reportó que no bebe ni fuma, el $33,5 \%$ solo bebe, el 1,8\% solo fuma y el 9,2\% bebe y fuma al mismo tiempo. El consumo combinado de tabaco y alcohol fue más frecuente en hombres $(18,3 \%)$. En el caso de las mujeres la prevalencia fue mayor entre los 45 a 59 años $(3,5 \%)$, las mestizas $(3,5 \%)$, aquellas con residencia en la región del trópico $(3,9 \%)$ y metropolitana $(3,0 \%)$, con nivel de educación superior $(5,7 \%)$, entre las solteras $(3,3 \%)$, entre las jubiladas $(6,2 \%)$ o autoempleadas (4\%); por el contrario, la prevalencia fue más baja entre las amas de casa $(1,3 \%)$ y entre las que viven en el Valle alto $(1,5 \%)$.

En el caso de los hombres, el consumo combinado fue más frecuente entre los 30 a 44 años (22,7\%), entre aquellos que viven en la región del trópico $(24,2 \%)$, con educación superior $(20,4 \%)$ y entre los autoempleados (20,7\%); por el otro lado la frecuencia fue más baja entre los ancianos (más de 60 años: $11,3 \%)$, los residentes del valle alto $(14,1 \%)$, entre los que no cursaron ningún nivel de educación formal (10,9\%), entre las categorías laborales de jubilados $(9,9 \%)$, estudiantes $(8,9 \%)$ y labores de hogar $(2,9 \%)$.

\section{Presión arterial elevada}

La prevalencia de PAE fue de $25 \%$, siendo más elevada en hombres $(30,7 \%)$ que en mujeres $(21,2 \%)$; la asociación entre PAE y el consumo combinado de tabaco y alcohol fue estadísticamente significativa $(\mathrm{X} 2=29,36 ; \mathrm{p}=<0,001)$. La prevalencia de PAE fue mayor entre aquellos que solo fuman $(36,1 \%)$, seguida de los que fuman y beben simultáneamente $(32,4 \%)$. La desagregación por razón de género resalta diferencias en la prevalencia de PAE entre aquellos que no beben ni fuman (Mujeres:19,4\%; Hombres:26,4\%), los que solo beben (Mujeres:24,2\%, Hombres:33,8\%) y aquellos que fuman y beben simultáneamente (Mujeres:27,4\%, Hombres:33,4\%). (figura 1).

La figura 1 describe la probabilidad de presentar PAE frente a los diferentes tipos de consumo de tabaco y alcohol en comparación a aquellos que no beben nifuman. La probabilidad de presentar PAE fue mayor y estadísticamente significativa en aquellos que solo fuman (OR:2,04-IC95\%:1,51-2,75), beben y fuman (OR:1,73 IC95\%:1,49-2,01) y aquellos que solo beben (OR:1,44 IC95\%: 1,31-1,58) en comparación con aquellos que no beben ni fuman; la desagregación de los OR por género muestra una diferencia marcada en el grupo de las personas que solo fuman siendo mayor el nivel de riesgo en mujeres (OR:2,21 IC95\%: 1,17-4,16) en comparación a los hombres (OR:1,58 IC95\%:1,12-2,24).

El modelo ajustado por edad para la evaluación descrita 


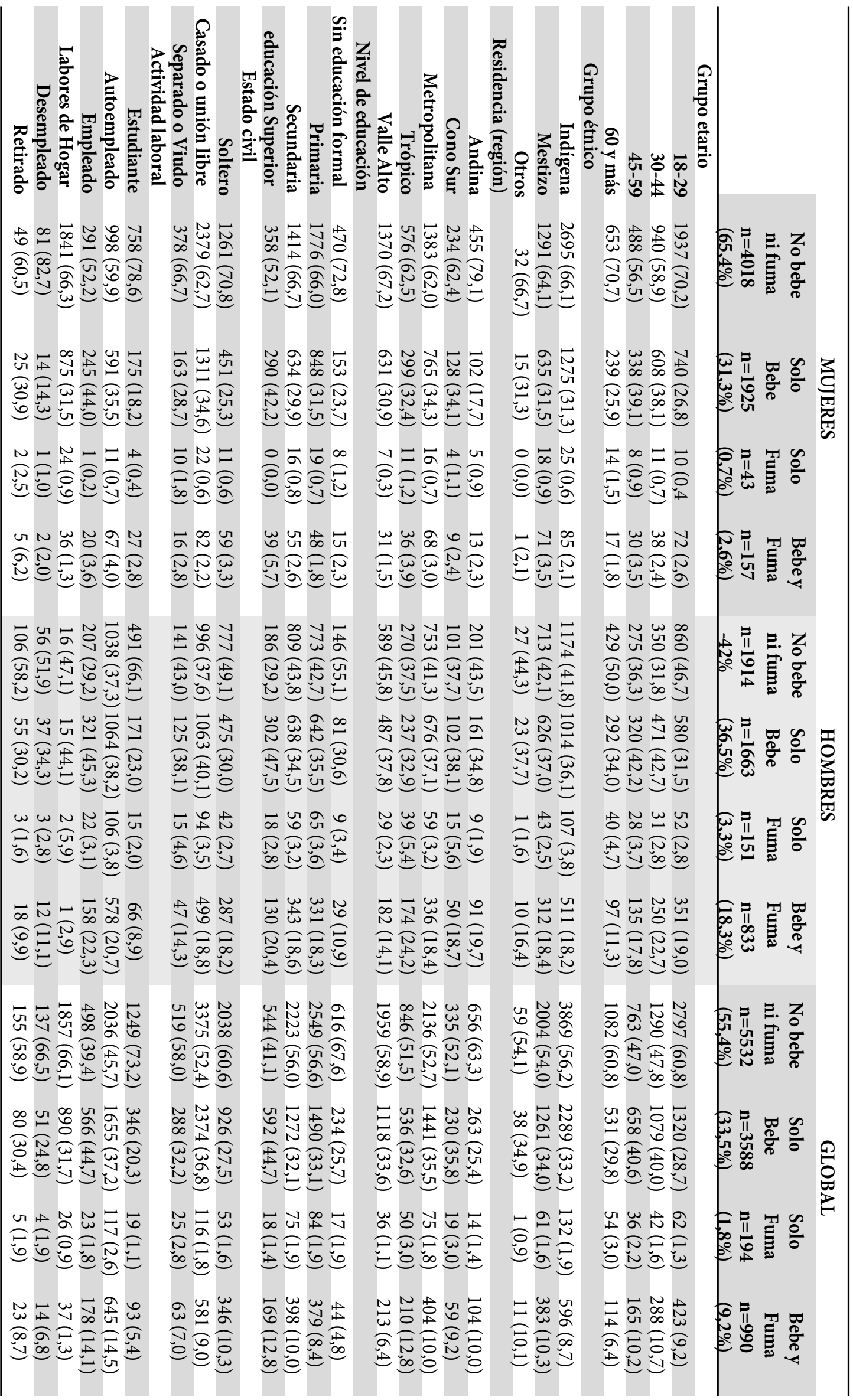




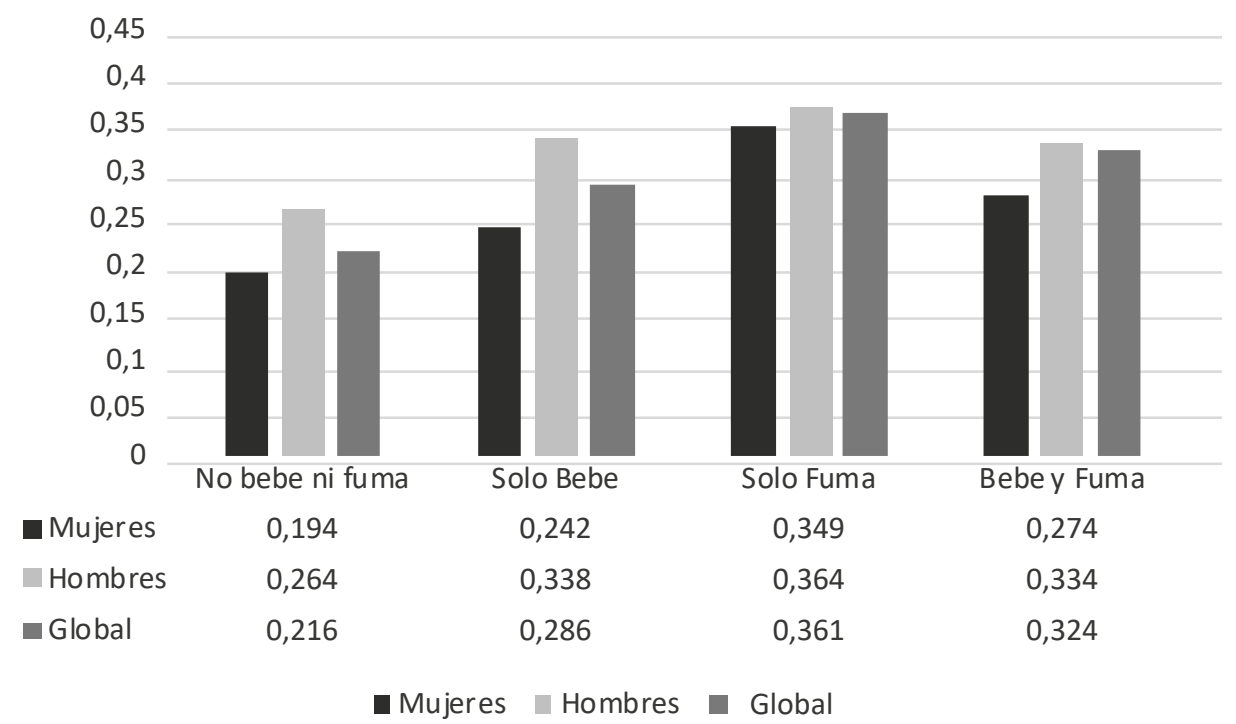

Figura 1: Distribución proporcional de la prevalencia de PAE según tipo de consumo de Tabaco y Alcohol desagregado por sexo.

en la figura 1, muestran un patrón similar, siendo la probabilidad de presentar PAE estadísticamente significativo para los 3 subgrupos de consumo de tabaco y alcohol, siendo mayor en el grupo de las personas que beben y fuman (OR:1,82 IC95\%:1,56-2,11) en especial las mujeres (OR:1,63 IC95\%:1,12-2,37) en comparación a los hombres. También se resalta el incremento de la probabilidad de presentar PAE conforme aumenta la edad, siendo la probabilidad más alta en las personas mayores de 60 años (OR:3,85 IC95\%:3,39-4,35) en comparación a las personas menores de 30 años; siendo mayor en mujeres (OR:4,70 IC95\%:3,95-5,58) en comparación a los hombres del mismo grupo etario.

Descomposición de Blinder-Oaxaca por referencia de género

La tabla 3 refleja la descomposición de las contribuciones absolutas y relativas de los factores sociodemográficos y conductuales evaluados por la metodología STEPS de la OMS, para explicar las diferencias por razón de género que en las prevalencias de PAE, consumo de tabaco y alcohol. Se observa una diferencia de $9,62 \%$ en la prevalencia de PAE entre hombres y mujeres; esta diferencia se explica en un $24,89 \%$ por los factores asociados evaluados en este estudio; siendo los factores más importantes para explicar esta brecha, las diferencias en el consumo de tabaco y alcohol $(63,99 \%)$ y grupo etario $(56,94 \%)$; observándose también una contribución negativa muy importante del tipo de actividad laboral $(-37,28 \%)$, en el sentido de reducir la brecha en la prevalencia de PAE entre hombre y mujeres.

En el caso de la prevalencia de consumo de tabaco, se observa una diferencia del 18,39\% entre hombres y mujeres, con una fracción explicada del $47,31 \%$ para los factores asociados contribuyentes evaluados en este estudio, siendo los más importantes para explicar esta brecha el consumo de alcohol $(54,04 \%)$ y tipo de actividad laboral (42,09\%).

La diferencia entre hombres y mujeres para la prevalencia de consumo de alcohol fue del 20,97\%; esta diferencia es
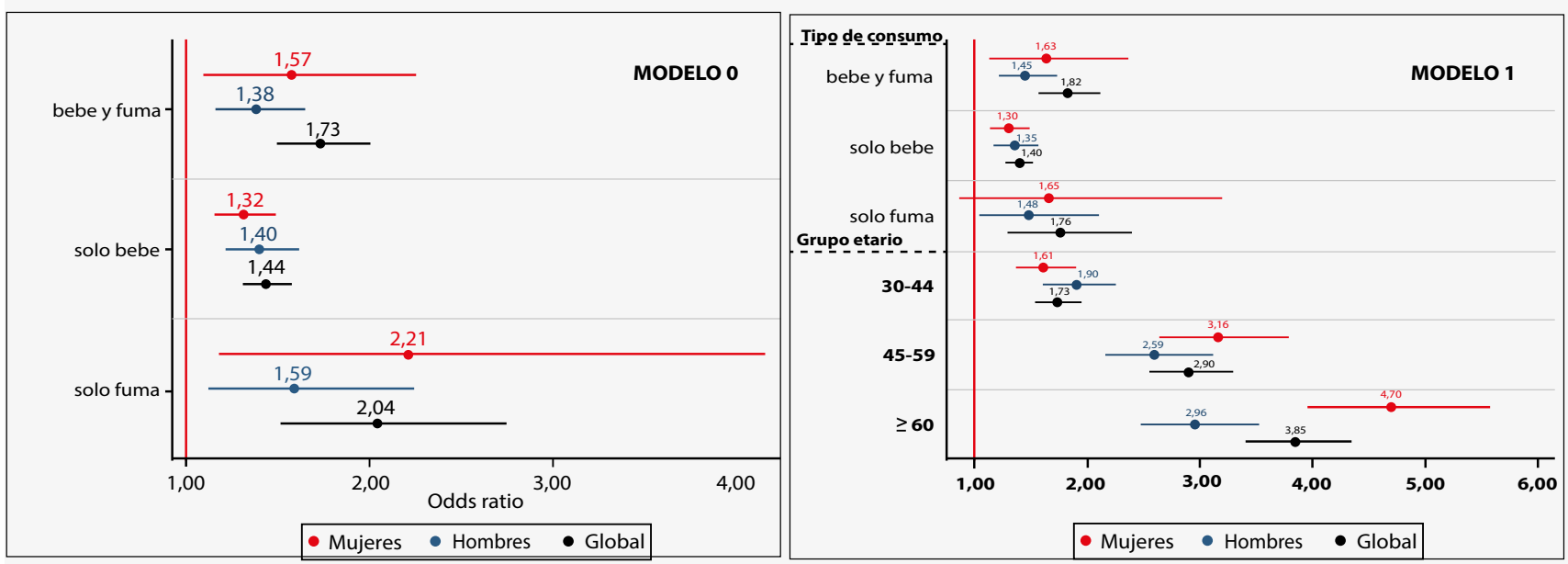

Figura 2: Niveles de Odds ratios para PAE por consumo de tabaco y alcohol 
Tabla 3: Descomposición de Blinder-Oaxaca por referencia de género para la PAE, consumo actual de tabaco y alcohol

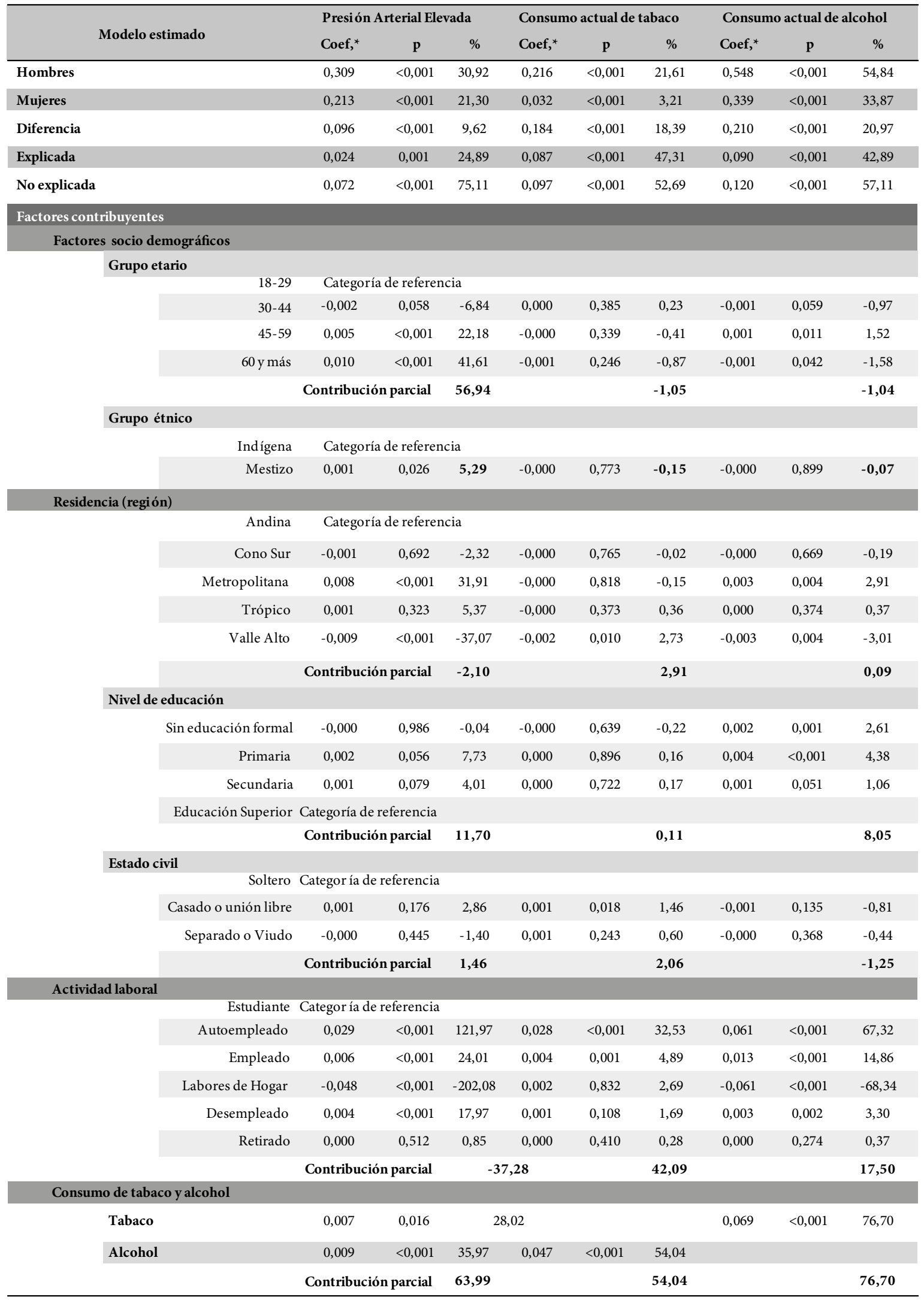

\section{NA: No Aplica}

(*) Coeficiente de regresión 
explicada en un $42,89 \%$ por las variables estudiadas, siendo los factores más importantes para explicar esta diferencia el consumo de tabaco $(76,70 \%)$ y el tipo de actividad laboral $(17,5 \%)$.

\section{Discusión}

Las asociación entre la presión arterial elevada con el consumo de tabaco y alcohol han sido descritos extensamente; sin embargo, en pocos casos se analiza su efecto combinado y su distribución en base a factores sociodemográficos. Por otro lado, las diferencias por razón de género también son revisadas en la mayoría de los estudios sobre PAE, tabaquismo y/o alcoholismo, sin embargo, son pocos los estudios que realicen una descomposición de los factores contribuyentes que expliquen estas diferencias entre ambos subgrupos poblacionales en todo el mundo ${ }^{16}$ y ninguno el contexto Latino Americano.

Nuestros resultados muestran una diferencia estadísticamente significativa entre hombres y mujeres para la prevalencia de tabaquismo y consumo nocivo del alcohol; estos son similares a los reportes internacionales de la $\mathrm{OMS}^{4,17}$, OPS $^{18}$ y estudios en América Latina ${ }^{19,20}$. Sin embargo, resalta que las prevalencias para tabaquismo actual en nuestro estudio $(11,1 \%)$ son menores al estimado para América Latina (Global:15,3\%), pero similar al del área Andina (11,7\%); muy por debajo al "estimado" para Bolivia en el mismo reporte (Global:23,7\%, Hombres: 30,5\%, Mujeres:17,1\%) ${ }^{18}$. En todos los casos se resalta una mayor prevalencia en los hombres, relacionado con los roles o estigma social sobre el tabaquismo en las mujeres, en quienes se reprocha su uso más que en los hombres, ya que socialmente se espera que las mujeres no fumen por su rol de cuidadoras del hogar, reflejándose en una incidencia menor de cáncer de pulmón en mujeres y es considerado un factor protector para el desarrollo de esta patología ${ }^{21}$. Sin embargo, el actual cambio de roles, como la inclusión de la mujer en actividades laborales anteriormente restringidas a hombres, alcanzar un nivel educativo y socioeconómico más alto, así como el control de la natalidad, se asocian a un incremento en la prevalencia de consumo de tabaco en las mujeres ${ }^{16,19,21}$ y dificultan su cesación ${ }^{22}$ que generalmente era a una edad más temprana en comparación a los hombres, como se reporta en nuestro estudio.

Observamos una situación similar para el consumo nocivo de alcohol, al igual que otros reportes internacionales ${ }^{4,6}$, 17 y locales ${ }^{19,23,24}$, nuestros resultados muestran una mayor prevalencia en los hombres; también se resalta entre nuestros hallazgos, que las mujeres con un nivel educativo superior, en edad laboral y con empleo formal o en autoempleo presentaron prevalencias más elevadas, al igual que otros reportes locales ${ }^{23,24}$ e internacionales ${ }^{4,17}$. Estos resultados apoyan la hipotesis de que un nivel sociocultural y posición económica más alta de las mujeres, las introduce a conductas nocivas consideradas propias de los hombres ${ }^{25,26}$, comenzando desde edades tempranas durante la adolescencia ${ }^{27}$, en especial en los países de América Latina.
La probabilidad de presentar hipertensión arterial fue mayor en las personas que solo fuman, solo beben, y aquellos que beben y fuman simultáneamente, en comparación a los individuos que no beben ni fuman, esta asociación generalmente es evaluada por separado y se la incluye dentro de las guías internacionales sobre el manejo de hipertensión arterial $^{7-9}$. Nuestros hallazgos resaltan que el uso aislado de tabaco presenta niveles de riesgo similares al uso combinado con el alcohol; siendo las mujeres el grupo que presentó valores OR más elevados en ambos tipos de consumo. Esta variación podría estar asociada a otros factores de riesgo no evaluados en el presente estudio, como el componente hormonal $^{28}$, la presencia de obesidad ${ }^{19,24}$, niveles de estrés ${ }^{29}$ y sedentarismo más altos en mujeres que en hombres ${ }^{19}$; debido a que comúnmente se ha reportado niveles de riesgo más altos para el desarrollo de hipertensión en hombres ${ }^{7}$.

El análisis de descomposición de Blinder-Oaxaca, nos brinda más luces sobre los factores que explican estas diferencias entre hombres y mujeres, que en el caso del consumo de tabaco y alcohol, las variables incluidas en este estudio explican en casi un 50\% las diferencias por razón de género, siendo los factores contribuyentes más importantes la actividad laboral y los factores conductuales, relacionado con la inclusión de la mujer en actividades fuera de las labores del hogar, así como un nivel educativo más alto, en concordancia de lo anteriormente analizado. Sin embargo, las diferencias de género en la prevalencia de PAE, solo se explicaron en un $25 \%$ por las variables incluidas en el presente estudio, siendo necesaria un análisis más profundo incluyendo otros factores de riesgo como la obesidad, sedentarismo y consumo de sal, que podrían incrementar esta fracción explicada. A pesar de lo anterior, se resalta que el grupo etario, así como el consumo de tabaco y alcohol, son los principales factores que contribuyen a explicar las diferencias de género en la prevalencia de PAE, refrendando todo lo descrito anteriormente.

Este estudio se basa en una muestra representativa de la población del departamento de Cochabamba, con información recopilada a través de un proceso estandarizado y validado por la OMS/OPS, sin embargo, la naturaleza transversal de los datos no permite inferencias causales. También se debe considerar que la información sobre el consumo de tabaco $\mathrm{y}$ alcohol pudo haberse ocultado por algunos participantes, por el temor a ser juzgados y/o se avergüenzan de responder afirmativamente; en consecuencia, su prevalencia podría haber sido subestimada. El análisis de descomposición de Oaxaca se puede ver como un método útil para identificar factores subyacentes a las desigualdades de salud; sin embargo, no puede ilustrar los procesos ni respaldar la inferencia causal en mayor grado que los modelos de regresión convencionales por la naturaleza transversal de nuestros datos.

Finalmente, nuestros hallazgos muestran que una baja prevalencia no necesariamente implica un bajo nivel de riesgo; existe diferencias de género en la interacción entre el tabaquismo y alcoholismo con la PAE, y a pesar de una baja prevalencia de los factores evaluados en las mujeres, la 
probabilidad de presentar PAE fue mayor, especialmente en aquellas mujeres que fuman; siendo el nivel de educación y el tipo de actividad laboral, los factores que más contribuyen a explicar estas diferencias.

Subvención: El Presente estudio fue ejecutado con fondos de la Agencia de Cooperación Sueca para el Desarrollo Internacional (ASDI; SIDA: Swedish International Development Cooperation Agency) de Cooperación para el
Desarrollo (ASDI) y el Programa de Formación Doctoral implementado por el Instituto de Investigaciones Biomédicas e Investigación Social de la Facultad de Medicina de la Universidad Mayor de San Simón (IIBISMEDUMSS). Los financiadores no tuvieron ninguna función en el diseño del estudio, la recopilación y el análisis de datos, la decisión de publicar o la preparación del manuscrito.

Conflicto de intereses: los autores declaran que no existe conflicto de intereses.

\section{Referencias bibliográficas}

1.- World Health Organization, Pan American Health Organization. Prevention and control of risk factors of noncommunicable diseases: State of the most cost-effective measures in Latin America. Geneva: World Health Organization; 2019. Available from: http://iris.paho.org/xmlui/bitstream/ handle/123456789/50833/9789275320662_spa. pdf? sequence $=1$ \&isAllowed $=y$.

2. Yeates K, Lohfeld L, Sleeth J, Morales F, Rajkotia Y, Ogedegbe O. A Global Perspective on Cardiovascular Disease in Vulnerable Populations. Can J Cardiol [Internet]. 2015; 31(9):[1081-93 pp.]. Available from: https://www.ncbi.nlm.nih. gov/pubmed/26321432

https://www.ncbi.nlm.nih.gov/pmc/articles/ PMC4787293/.

3. World Health Organization. Health Situation in the Americas: Core Indicators 2017. . Washington, D.C., United States of America, 2017. Available from: http://www.paho.org/data/index.php/en/ indicators.html.

4. World Health Organization. World health statistics 2017: monitoring health for the SDGs, sustainable development goals 2017. Available from: http://apps.who.int/iris/bitstream/ha ndle/10665/255336/9789241565486-eng. pdf? sequence $=1$.

5. Fernández Moreno SY. Postura de la Red Latinoamericana de Género y Salud Colectiva de ALAMES frente a la cobertura universal de salud. Revista Cubana de Salud Pública [Internet]. 2015; 41:[335-56 pp.]. Available from: https://www.scielosp.org/scielo. php?pid=S0864-34662015000200012\&script=sci arttext\&tlng=en\#ModalArticles.

6. World Health Organization. NonCommunicable Diseases Country Profiles 2018. Geneva Switzerland: World Health Organization/Pan American Health Organization; 2018 [cited 2019. Available from: https://www.who.int/nmh/ publications/ncd-profiles-2018/en/.

7. Whelton PK, Carey RM, Aronow WS, Casey DE, Collins KJ, Himmelfarb CD, et al. 2017 ACC/ AHA/AAPA/ABC/ACPM/AGS/APhA/ASH/ ASPC/NMA/PCNA guideline for the prevention, detection, evaluation, and management of high blood pressure in adults: a report of the American College of Cardiology/American Heart Association Task Force on Clinical Practice Guidelines. Journal of the American College of Cardiology [Internet]. 2018; 71(19):[e127-e248 pp.]. Available from: http://www.onlinejacc.org/ content/accj/71/19/e127.full.pdf.
8. Leung AA, Nerenberg K, Daskalopoulou SS, McBrien K, Zarnke KB, Dasgupta K, et al. Hypertension Canada's 2016 Canadian Hypertension Education Program Guidelines for Blood Pressure Measurement, Diagnosis, Assessment of Risk, Prevention, and Treatment of Hypertension. Canadian Journal of Cardiology [Internet]. 2016 2016/05/01/; 32(5):[569-88 pp.]. Available from: http://www.sciencedirect.com/ science/article/pii/S0828282X16001926.

9. Bakris G, Ali W, Parati G. ACC/AHA Versus ESC/ESH on Hypertension Guidelines: JACC Guideline Comparison. Journal of the American College of Cardiology [Internet]. 2019 2019/06/18/; 73(23):[3018-26 pp.]. Available from: http://www.sciencedirect.com/science/ article/pii/S0735109719348879.

10. Kim S, Chang Y, Kang J, Cho A, Cho J, Hong YS, et al. Relationship of the Blood Pressure Categories, as Defined by\&\#xa0;the ACC/ AHA\&\#xa0;2017 Blood Pressure Guidelines, and the Risk of\&\#xa0;\&\#xa0;Development of Cardiovascular Disease in Low\&\#x2010;Risk Young\&\#xa0;\&\#xa0;Adults: Insights From a Retrospective Cohort of Young\&\#xa0;Adults. Journal of the American Heart Association [Internet]. 2019; 8(11):[e011946 p.]. Available from: https://www.ahajournals.org/doi/ abs/10.1161/JAHA.119.011946.

11. Sen B. Using the oaxaca-blinder decomposition as an empirical tool to analyze racial disparities in obesity. Obesity [Internet]. 2014; 22(7):[1750-5 pp.]. Available from: http:https://doi.org/10.1002/ oby. 20755.

12. Jann B. A Stata implementation of the BlinderOaxaca decomposition2008; 5. Available from: https://ideas.repec.org/p/ets/wpaper/5.html.

13. World Health Organization. WHO STEPS surveillance manual: the WHO STEPwise approach to chronic disease risk factor surveillance 2005. Available from: http://apps.who.int/iris/ bitstream/handle/10665/43376/9241593830_eng. pdf;jsessionid=FDC45147001C32D84F6A286406 $48 \mathrm{E} 90 \mathrm{D}$ ? sequence $=1$.

14. Instituto Nacional de Estadística Bolivia. Estado Plurinacional de Bolivia Censo Nacional de Población y Vivienda: Cochabamba. In: INE, editor. La Paz-Bolivia.2012. p. 30-5.

15. Lilliefors HW. On the Kolmogorov-Smirnov Test for Normality with Mean and Variance Unknown. Journal of the American Statistical Association [Internet]. 1967 1967/06/01; 62(318):[399-402 pp.]. Available from: http:// amstat.tandfonline.com/doi/abs/10.1080/016214

\subsection{7 .10482916}

16. Bauer T, Göhlmann S, Sinning M. Gender differences in smoking behavior. Health Economics [Internet]. 2007; 16(9):[895-909 pp.]. Available from: https://onlinelibrary.wiley.com/ doi/abs/10.1002/hec.1259.

17. World Health Organization WHO, Unit WHOMoSA. Global status report on alcohol and health, 2014: World Health Organization; 2014. Available from: http://apps.who.int/iris/ bitstream/handle/10665/112736/9789240692763 eng.pdf? sequence $=1$.

18. Organización Panamericana de la Salud/Organización Mundial de la Salud. Evidencia e Inteligencia para la Acción en Salud / Análisis de Salud, Métricas y Evidencia: Situación de Salud en las Américas: Indicadores Básicos 2018. Washington, D.C., Estados Unidos de America, 2018 [cited 2019 09-032019]. Available from: http://iris.paho.org/ xmlui/bitstream/handle/123456789/49511/ In d i c a d ores B a si cos 2018 _ s p a pdf? sequence $=2$ \&isAllowed $=y$.

19. Mamani-Ortiz Y, San Sebastián M, Armaza AX, Luizaga JM, Illanes DE, Ferrel $\mathrm{M}$, et al Prevalence and determinants of cardiovascular disease risk factors using the WHO STEPS approach in Cochabamba, Bolivia. BMC Public Health [Internet]. 2019 June 20; 19(1):[786 p.]. Available from: https://doi.org/10.1186/s12889019-7064-y.

20. Paz-Ballesteros WC, Zavala-Arciniega L, Gutiérrez-Torres DS, Ponciano-Rodríguez G, Reynales-Shigematsu LM. Evaluación de la dependencia física y psicológica al tabaco en fumadores mexicanos adultos, Encodat 2016. 2019 [Internet]. 2019 2019-01-10; 61(2, Mar-Abr):[11 p.]. Available from: http://www.saludpublica.mx/ index.php/spm/article/view/9858.

21. Borràs JM. La perspectiva del género en el cáncer: una visión relevante y necesaria. 2015 [Internet]. 2015 2015-06-30; 191(773). Available from: http://arbor.revistas.csic.es/index.php/ arbor/article/view/2034.

22. Smith PH, Bessette AJ, Weinberger AH, Sheffer CE, McKee SA. Sex/gender differences in smoking cessation: A review. Preventive Medicine [Internet]. 2016 2016/11/01/; 92:[135-40 pp.]. Available from: http://www.sciencedirect.com/ science/article/pii/S009174351630189X.

23. Abasto Gonzales DS, Mamani Ortiz Y, Luizaga Lopez JM, Pacheco Luna S, Illanes Velarde DE. Factores de riesgo asociados 
al síndrome metabólico en conductores del transporte público en Cochabamba-Bolivia. Gaceta Médica Boliviana [Internet]. 2018; 41:[4757 pp.]. Available from: http://www.scielo.org. bo/scielo.php?script $=$ sci_arttext\&pid $=\mathrm{S} 1012$ 29662018000100010\&nrm=iso.

24. Mamani Ortiz Y, Armaza Cespedes A, Medina Bustos M, Luizaga Lopez JM, Abasto Gonzales DS, Argote Omonte M, et al. Caracterización del perfil epidemiológico del síndrome metabólico y factores de riesgo asociados. Cochabamba, Bolivia. Gaceta Médica Boliviana [Internet]. 2018; 41:[24-34 pp.]. Available from: http://www.scielo. org.bo/scielo.php?script=sci_arttext\&pid=S101229662018000200007\&nrm=iso.

25. Peltzer K, Pengpid S. Concurrent Tobacco Use and Binge Drinking Among University Students in 30 Countries in Africa, Asia, Latin America, and the Caribbean. International Journal of Mental Health and Addiction [Internet]. 2018 February 01; 16(1):[164-74 pp.]. Available from: https://doi.org/10.1007/s11469-017-9850-z.

26. Waldron I, Bratelli G, Carriker L, Sung W-C, Vogeli C, Waldman E. Gender differences in tobacco use in Africa, Asia, the Pacific, and Latin America. Social Science \& Medicine [Internet]. 1988 1988/01/01/; 27(11):[1269-75 pp.]. Available from: http://www.sciencedirect.com/science/ article/pii/0277953688903577.

27. Melotti R, Heron J, Hickman M, Macleod J, Araya R, Lewis G. Adolescent Alcohol and Tobacco Use and Early Socioeconomic Position:
The ALSPAC Birth Cohort. Pediatrics [Internet]. 2011; 127(4):[e948-e55 pp.]. Available from: https://pediatrics.aappublications.org/content/ pediatrics/127/4/e948.full.pdf.

28. Reckelhoff JF. Gender differences in hypertension. Current Opinion in Nephrology and Hypertension [Internet]. 2018 //; 27(3):[176-81 pp.]. Available from: https:// www.ingentaconnect.com/content/wk/ $\mathrm{mnh} / 2018 / 00000027 / 00000003 /$ art00008. https:/ doi.org/10.1097/MNH.0000000000000404.

29. Di Pilla M, Bruno RM, Taddei S, Virdis A. Gender differences in the relationships between psychosocial factors and hypertension. Maturitas [Internet]. 2016 2016/11/01/; 93:[58-64 pp.]. Available from: http://www.sciencedirect.com/ science/article/pii/S0378512216301359. 